\title{
DEVELOPABLE SURFACES IN EUCLIDEAN SPACE
}

\author{
VITALY USHAKOV
}

(Received 5 January 1997; revised 3 November 1997)

\author{
Communicated by R. Bartnik
}

\begin{abstract}
The classical notion of a two-dimensional developable surface in Euclidean three-space is extended to the case of arbitrary dimension and codimension. A collection of characteristic properties is presented. The theorems are stated with the minimal possible integer smoothness. The main tool of the investigation is Cartan's moving frame method.
\end{abstract}

1991 Mathematics subject classification (Amer. Math. Soc.): primary 53A07.

Keywords and phrases: Developable surface, planar point, ruled surface, flat metric, point codimension of a surface, local codimension of a surface, affinely stable immersion.

\section{Classical developable surfaces and terminology}

In this paper by a surface is meant a submanifold in Euclidean space considered locally, that is, in a neighbourhood of a point.

In classical differential geometry a developable surface $F^{2} \subset E^{3}$ is a surface which can be isometrically 'developed' onto the plane, that is, there exists a bending of $F^{2}$ onto $E^{2}$ [HC, Section 30]. Such surfaces possess a rich collection of properties. Namely, a developable surface

(1) has vanishing Gaussian curvature;

(2) is a ruled surface whose tangent plane is stable along the rulings (generators) (following Euler such a surface is called a torse);

(3) is an envelope of a 1-parameter family of planes;

(4) allows isometric bendings such that all rectilinear rulings remain straight lines (the original bending onto the plane can be obtained in this way).

(C) 1999 Australian Mathematical Society 0263-6115/99 \$A2.00+0.00 
It is remarkable that each of these properties is characteristic (with some stipulations, however). The precise statement of the equivalence of (1) and (2) is in [HW1, Theorems 4, 5; HW2, Theorems 13-15; HN, pp. 916-920]. The rest of the equivalences can be found in [Sp1, pp. 205-213, 262, 349-367]. Besides that there is

THEOREM 0 (The classical classification theorem [Sp1, p. 353]). An analytic connected developable surface $F^{2} \subset E^{3}$ is either a plane, cylinder, cone, or the tangent developable of some curve.

The tangent developable of a curve $\gamma \subset E^{3}$ is the surface consisting of the points of the tangent lines to $\gamma$. In order to avoid the nuisance noticed by Spivak [Sp1, p. 353] (the surface can be made up of two tangent developables joined together along a line belonging to neither) it is sufficient to allow $\gamma$ to be a curve from the projective point of view, that is, $\gamma$ can pass through a point at infinity (the line mentioned above is just the tangent to $\gamma$ at the point at infinity). It is worth noting that this condition is quite natural, for the class of developable surfaces is projectively invariant.

This paper aims to single out a class of many-dimensional surfaces most fully inheriting the properties of the classical developable surfaces $F^{2} \subset E^{3}$. Evidently, the same definition without any difficulty is transferred to the case of hypersurfaces: a hypersurface $F^{l} \subset E^{l+1}$ allowing a development (isometry) onto $l$-dimensional plane

(1) has the flat metric (vanishing sectional curvature);

(2) is an $(l-1)$-ruled surface (for every point there exists an $(l-1)$ - dimensional plane lying on the surface and passing through this point), and the tangent plane to the surface is stable along the rulings;

(3) is an envelope of a 1-parameter family of $l$-planes;

(4) allows isometric bendings which keep the rectilinear rulings.

And again, these properties are characteristic (to a certain extent).

However, with increase in the codimension, the same definition appears to be meaningless: the surfaces isometric to the plane still possess the flat metric, yet the ruling structure is lost (see the Clifford torus in Section 4). Therefore, in addition, one has to enforce the linear structure (or another useful property). Thus, a developable surface (from our point of view) is an $(l-1)$-ruled surface, possessing the flat metric. In this paper, however, we prefer to introduce another, more traditional definition which uses the nullity space of the second fundamental form of the surface. Naturally, this definition is equivalent to the one just given (Theorem 2) as well as to several others (Theorems 1-5). Besides, it worth noting that for our developable surfaces there exists a classification theorem quite similar to the classical one [U1].

Before going into precise definitions and statements, we have to linger a little over one long-lasting delusion regarding the term 'developable surfaces'. The matter is 
that for many decades various authors have been studying one and the same object, rarely suspecting that it had been already named and much had been known about it. This object is a many-dimensional generalisation of a torse, that is, a $k$-ruled surface $F^{l} \subset E^{n}$ with tangent plane stable along the $k$-rulings. (These surfaces appear to be of interest in many places; in particular, they and only they provide the complete bending hypersurfaces $F^{l} \subset E^{l+1}[D G]$; in this situation the dimension of the rulings is $k=l-2$.) So, these surfaces are also-known-as:

(1) surfaces of constant rank (Yanenko [Ya1], Akivis [A], Dajczer and Gromoll [DG]);

(2) tangentially degenerate surfaces (Ryzhkov [R], Akivis and Ryzhkov [AR]);

(3) $k$-developable surfaces (Shefel' [Sh], Toponogov and Shefel' [TSh], Fischer and $\mathrm{Wu}[\mathrm{FW}], \mathrm{Wu}[\mathrm{W}]$;

(4) strongly k-parabolic surfaces (Borisenko [B]);

(5) surfaces of constant relative nullity index (the majority of English authors beginning with Chern and Kuiper [CK]).

It seems no one has thought of naming such a surface a $k$-torse (the idea sounds not so bad!). In this paper I will use the term surface of constant nullity, which is short for the name given by Chern and Kuiper.

The origin of the term 'surfaces of constant rank' is quite explainable due to the following simple fact: the relative nullity index equals $v$ if and only if the rank of the Gauss map $F^{l} \rightarrow G(l, n)$ equals $l-v$ (the nullity space $\Delta$ is the kernel of the differential of the Gauss map); the precise definitions are given in the next section. It seems 'surfaces of constant rank' were introduced by Yanenko in 1949 [Ya1] (in the non-trivial case of codimension greater than 1; for hypersurfaces the Gauss map is rather simple and the term 'constant rank' was in use already in the end of the last century).

The term 'tangentially degenerate surface' appeals to the special ruling structure of the surface ( $k$-torse).

However, the name ' $k$-developable surface' (for a surface of constant nullity $k$ ) is totally inappropriate for the following simple reason: a surface $F^{l} \subset E^{l+p}$ of constant nullity $k$, as a rule, possesses essentially non- flat metric (when $k<l-1$ ) and therefore an isometry of such a surface onto the plane is out of question. Let us recall: 'a development of one surface on another is the very classical name of an isometry' [Sp1, p. 212], hence 'developable' means the existence of an isometry between the surface and the plane.

This delusion is rather widespread-we only observe that [TSh] is a paper in Encyclopaedia of Mathematics. The cause of the delusion can be traced: it is generated by a certain confusion reigning in the majority of the modern textbooks regarding the classical developable surfaces $F^{2} \subset E^{3}$. Originally, many years ago they used both 
torses and developable surfaces, which are defined differently, but appear to be almost (but not entirely!) the same. Gradually the term 'torse' was forced out (Encyclopaedia of Mathematics regards them to be identical: same entry for both). Moreover, the authors seeking simplicity began to define developable surfaces as 'ruled surfaces with tangent plane constant along the rulings'. Such a definition, apart from being inconsistent with the word used, led to the above stated erroneous generalisation for the many-dimensional case. Besides that, it produced absurdities like 'a surface of vanishing curvature which is not developable', that is, 'a surface of vanishing curvature which is not isometric to the plane' (locally!), for more details see [U2].

To conclude the terminological discussion we observe that developable surfaces concerned in this paper are $l$-dimensional surfaces of constant nullity $l-1$, or surfaces of constant rank 1 .

\section{Main definition}

The relative nullity index $v$ of a surface $F^{l} \subset E^{n}$ at a point $Q$ is the dimension of the nullity space

$$
\Delta=\left\{x \in T_{Q} F^{l} \mid A(x, \cdot)=0\right\},
$$

where $A$ is the vector-valued second fundamental form $A: T_{Q} F^{l} \times T_{Q} F^{l} \rightarrow T_{Q}^{\perp} F^{l}$; here $T_{Q} F^{l}$ and $T_{Q}^{\perp} F^{l}$ are the tangent and normal spaces to $F^{l}$ at $Q$ [CK].

We call a surface $F^{l} \subset E^{n}$ a developable surface if for every point $Q \in F^{l}$ the relative nullity index satisfies $v=l-1$. In other words, for any $Q \in F^{l}$ one can choose an orthonormal frame $\left\{e_{i}\right\}_{1}^{l}$ in the tangent space $T_{Q} F^{l}$ such that $A_{l l} \neq 0$, but all the other vectors $A_{i j}$ are zero (here $A_{i j}=A\left(e_{i}, e_{j}\right)$ ).

REMARK. Quite often it seems reasonable to allow a developable surface to have not only points with $v=l-1$, but also planar points $(v=l)$. The presence of planar points, however, may destroy the rulings and drastically reduce the smoothness of the surface [U2]. For this reason we prefer initially to consider the developable surfaces without planar points and in Section 6 briefly discuss what happens with each theorem if the planar points are taken into consideration.

\section{Developable surfaces as ruled surfaces}

A surface $F^{l} \subset E^{n}$ is a $k$-ruled surface if it is made up of $k$-planes in such a way that for every point of the surface there is the unique $k$-plane passing through this 
point and belonging to the surface; these planes are called the rectilinear rulings of $F^{l}$. A $k$-ruled surface has a standard parametrization:

$$
r\left(u^{1}, \ldots, u^{l}\right)=\rho\left(u^{k+1}, \ldots, u^{l}\right)+\sum_{i=1}^{k} u^{i} \cdot a_{i}\left(u^{k+1}, \ldots, u^{l}\right)
$$

the $(l-k)$-dimensional surface $\rho\left(u^{k+1}, \ldots, u^{l}\right)$ is a directrix of $F^{l}$; the linear hull span $\left(a_{1}, \ldots, a_{k}\right)$ gives the direction of a ruling. Henceforth we will use Einstein's summation convention, so that the above equation turns into $r=\rho+u^{i} a_{i}$.

THEOREM 1. A $C^{2}$ smooth surface $F^{l} \subset E^{n}$ is a developable surface if and only if $F^{l}$ is $(l-1)$-ruled (but not $l$-ruled anywhere, that is, without planar points) and the tangent planes are stable along the rulings.

The essential part of Theorem 1 (the necessity) is a particular case of a well- known fact (a surface $F^{l} \subset E^{n}$ of constant nullity $k$ is $k$-ruled and the tangent planes are stable along the rulings) which has been proved in many places. The final version with the lowest possible integer smoothness- $C^{2}$ was accomplished by Hartman $[\mathrm{H}$, Lemma 3.1]. We give a simpler proof for a $C^{4}$ surface adapting one from [Ya2]. Our proof can also be refined to the $C^{2}$ version [U3].

It should be also mentioned here, that the Hartman's result allows a generalisation for Riemannian manifolds: the $k$-nullity distribution is integrable and has totally geodesic leaves; see [KN, Note 16, pp. 347-349].

REMARK. The theorem states that a $C^{2}$ smooth developable surface $F^{l} \subset E^{n}$ possesses a standard parametrization for ruled surfaces. Such a parametrization is $C^{1}$ smooth [HW2, Theorem 14 for $F^{2} \subset E^{n} ; \mathrm{H}$, p. 97 for $F^{l} \subset E^{n}$ ], but might be not $C^{2}$ smooth; more precisely, $\rho$ can be chosen $C^{2}$, but $a_{i} \notin C^{2}$ in the general situation [HW2, Theorem 15]; see also [U2] for a detailed discussion.

Theorem 1 has the following simple corollary.

THEOREM 2. A $C^{2}$ smooth surface $F^{l} \subset E^{n}$ is a developable surface if and only if $F^{l}$ is $(l-1)$-ruled (without planar points) and its metric is flat.

PROOF OF THEOREM 1 (sufficiency). Given a $C^{2}$ smooth $(l-1)$-ruled surface $F^{l} C$ $E^{n}$, one can choose a $C^{2}$ smooth orthonormal frame $\left\{e_{A}\right\}_{1}^{n}$ such that $\operatorname{span}\left\{e_{i}\right\}_{1}^{l-1}$ gives the direction of the ruling, and span $\left\{e_{\alpha}\right\}_{l+1}^{n}$ gives $T^{\perp} F^{l}$. Then the radius vector of $F^{l}$ can be written as

$$
r\left(u^{1}, \ldots, u^{l}\right)=\rho\left(u^{l}\right)+u^{i} e_{i}\left(u^{l}\right) \quad(1 \leq i \leq l-1),
$$


where we can assume without loss of generality $\dot{\rho}=e_{l}$.

Then the values of the second fundamental form satisfy $A_{i j}=0(1 \leq i, j \leq l-1)$. On the other hand the condition of the tangent plane being stable along the rulings implies $A_{i l}=0(1 \leq i \leq l-1)$ :

(2) $d_{e_{i}}\left(T F^{l}\right) \subset T F^{l} \Longleftrightarrow\left\{\begin{array}{l}0=\left\langle d_{e_{i}} e_{j}, e_{\alpha}\right\rangle=A_{i j}^{\alpha} \\ 0=\left\langle d_{e_{i}} e_{l}, e_{\alpha}\right\rangle=A_{i l}^{\alpha}\end{array} \quad\left(\begin{array}{c}1 \leq i, j \leq l-1 \\ l+1 \leq \alpha \leq n\end{array}\right)\right.$

(here $\langle\cdot, \cdot\rangle$ is the scalar product in $E^{n}$ and $d_{e_{i}}$ denotes the derivative in the direction of $e_{i}$ ). The final condition $A_{l l} \neq 0$ is implied by the absence of planar points.

PROOF OF THEOREM 1 (necessity). Let $F^{l}$ be a $C^{4}$ developable surface.

Step 1. Construction of a $C^{2}$-smooth orthonormal frame, satisfying the condition of the definition of a developable surface. As $F^{\prime}$ is $C^{4}$ smooth, the tangent bundle $T F^{l}$ is $C^{3}$ smooth. On $F^{l}$ one can choose a $C^{3}$ smooth orthonormal frame $\left\{\tilde{e}_{A}\right\}_{1}^{n}$ with $\left\{\tilde{e}_{i}\right\}_{1}^{l} \subset T F^{l}$. Let $\tilde{e}_{\alpha}$ be an arbitrary non- trivial normal $\left(A^{\alpha} \neq 0\right)$. The projection of the second fundamental form onto this normal $A^{\alpha}=\left\langle A, e_{\alpha}\right\rangle: T_{Q} F^{l} \times T_{Q} F^{l} \rightarrow \mathbb{B}$ becomes a real-valued bilinear symmetric form. There is the selfadjoint Weingarten operator $\tilde{A}: T_{Q} F^{l} \rightarrow T_{Q} F^{l}$ associated with $A^{\alpha}$ and acting by defining $\tilde{A}(e)$ to be the unique vector satisfying: $\langle\tilde{A}(e), x\rangle=A^{\alpha}(e, x)$ for all $x \in T_{Q} F^{l}$ (here $\langle\cdot, \cdot\rangle$ is the scalar product in $\left.T_{Q} F^{l}\right)$. Since the relative nullity index $v$ is $l-1$, the image $\tilde{A}\left(T_{Q} F^{l}\right)$ has dimension 1. Choosing at every point $Q \in F^{l}$ a unit vector $e_{l}$ in $\tilde{A}\left(T_{Q} F^{l}\right)$ one obtains a $C^{2}$ smooth vector field on $T F^{l}\left(T F^{l}\right.$ is $C^{3}$ smooth; $A, A^{\alpha}, \tilde{A}$ are $C^{2}$ smooth). Now one can construct the required $C^{2}$ smooth orthonormal frame $\left\{e_{i}\right\}_{1}^{l}$ in $T F^{l}$ (by rotation of the initial $\left\{\tilde{e}_{i}\right\}_{1}^{l}$, for instance). For this frame $A_{i j}=A_{i l}=0(1 \leq i, j \leq l-1)$, $A_{l l} \neq 0$.

Expanding the differentials of the frame by the frame itself

$$
\left\{\begin{array}{l}
d e_{i}=\psi_{i}^{j} e_{j}+h_{i}^{\alpha} e_{\alpha} \\
d e_{\alpha}=h_{\alpha}^{i} e_{i}+\kappa_{\alpha}^{\beta} e_{\beta}
\end{array} \quad\left(\begin{array}{c}
1 \leq i, j \leq l \\
l+1 \leq \alpha, \beta \leq n
\end{array}\right),\right.
$$

the definition of a developable surface becomes equivalent to the following equations on the mixed forms $h_{i}^{\alpha}$ :

$$
\left\{\begin{array}{l}
h_{i}^{\alpha}=A_{i j}^{\alpha} \cdot \omega^{j}=0 \\
h_{l}^{\alpha}=A_{l l}^{\alpha} \cdot \omega^{l} \neq 0
\end{array} \quad(1 \leq i \leq l-1, \quad 1 \leq j \leq l, \quad l+1 \leq \alpha \leq n),\right.
$$

where the $\omega^{i}$ are the dual forms for the orthonormal frame $e_{i}$.

Step 2. The surface $F^{l}$ is $(l-1)$-ruled. We intend to prove that the distribution $\Delta=\operatorname{span}\left\{e_{i}\right\}_{1}^{l-1}$ is integrable and its leaves are totally geodesic in the ambient space $E^{n}$, that is, are planes. 
Let us demonstrate that the integrability of $\Delta$ can be analytically expressed as

$$
\Gamma_{i j}^{l}=\Gamma_{j i}^{l} \quad(1 \leq i, j \leq l-1),
$$

and the leaves of $\Delta$ are totally geodesic if and only if

$$
\Gamma_{i j}^{l}=0 \quad(1 \leq i, j \leq l-1),
$$

where the $\Gamma_{i j}^{k}$ are the coefficients in the expansion of the connection forms $\psi$ by the forms $\omega$ :

$$
\psi_{i}^{k}=\Gamma_{i j}^{k} \omega^{j} \quad(1 \leq i, j, k \leq l) .
$$

Due to Frobenius' theorem the integrability of $\Delta=\operatorname{span}\left\{e_{i}\right\}_{1}^{l-1}$ is tantamount to $\left[e_{i}, e_{j}\right]=d_{e_{i}} e_{j}-d_{e_{j}} e_{i} \in \Delta(1 \leq i, j \leq l-1)$, or

$$
\begin{cases}\left\langle d_{e_{i}} e_{j}-d_{e_{j}} e_{i}, e_{l}\right\rangle=0 & 1 \leq i, j \leq l-1 \\ \left\langle d_{e_{i}} e_{j}-d_{e_{j}} e_{i}, e_{\alpha}\right\rangle=0 & l+1 \leq \alpha \leq n .\end{cases}
$$

The first equation can be written as $\psi_{j}^{l}\left(e_{i}\right)=\psi_{i}^{l}\left(e_{j}\right)$, which with the expansion (7) gives (5). The second equation is valid automatically since the nullity distribution $\Delta$ lies in the integrable distribution $T F^{l}$ (or, what is the same, as the second fundamental form is a symmetric bilinear form).

The leaves of $\Delta$ are totally geodesic if and only if they are autoparallel [Sp1, p. 35], that is, $d_{e_{i}} e_{j} \in \Delta(1 \leq i, j \leq l-1)$. This condition splits into two equations. The first one $\left\langle d_{e_{i}} e_{j}, e_{\alpha}\right\rangle=0$ is valid automatically since $\left\langle d_{e_{i}} e_{j}, e_{\alpha}\right\rangle=h_{j}^{\alpha}\left(e_{i}\right)=0$ from (4). The second equation $\left\langle d_{e_{i}} e_{j}, e_{l}\right\rangle=0$ leads to (6): $0=\left\langle d_{e_{i}} e_{j}, e_{l}\right\rangle=\psi \psi_{j}^{l}\left(e_{i}\right)=\Gamma_{j i}^{l}$.

Since (6) implies (5) one only needs to obtain (6) in order to complete Step 2. These equations are a part of the Codazzi-Mainardi equations for $F^{l} \subset E^{n}$. Indeed, the Codazzi-Mainardi equations [Sp2, p. 63] are

$$
d h_{i}^{\alpha}+h_{k}^{\alpha} \wedge \psi_{i}^{k}+\kappa_{\beta}^{\alpha} \wedge h_{i}^{\beta}=0 \quad\left(\begin{array}{c}
1 \leq i, k \leq l \\
l+1 \leq \alpha, \beta \leq n
\end{array}\right)
$$

Considering them for $1 \leq i \leq l-1$ and using (4), one has $0=h_{k}^{\alpha} \wedge \psi_{i}^{k}=A_{l l}^{\alpha} \omega^{l} \wedge \psi_{i}^{l}$, whence with (7) $0=\omega^{l} \wedge \psi_{i}^{l}=\Gamma_{i j}^{l} \omega^{l} \wedge \omega^{j}$, which gives exactly (6).

Step 3. The tangent planes are stable along the rulings. From (4) $A_{i j}=A_{i l}=0$ $(1 \leq i, j \leq l-1)$. Using (2) one obtains the statement required.

PROOF OF THEOREM 2. This theorem reduces to the previous one due to the following fact: for an $(l-1)$-ruled surface $F^{l} \subset E^{n}$ the condition of the tangent planes being stable along the rulings is equivalent to the flatness of the metric. Indeed, given 
an $(l-1)$-ruled surface (1), one has $A_{i j}=\operatorname{pr}_{T^{1} F^{\prime}}\left(r_{i j}\right)=0(1 \leq i, j \leq l-1)$. Then the tangent planes are stable if and only if $A_{i l}=0(1 \leq i \leq l-1)$ (see (2)). The surface has the flat metric if and only if $0=R_{i l i l}=A_{i i} \cdot A_{l l}-\left(A_{i l}\right)^{2}=-\left(A_{i l}\right)^{2}$ $(1 \leq i \leq l-1)$ (all other components of the curvature tensor already vanish due to $A_{i j}=0$ ).

\section{Developable surfaces as surfaces of point codimension 1}

For a surface $F^{l} \subset E^{n}$ the first normal space of a point $Q \in F^{l}$ is the orthogonal complement in $T_{Q}^{\perp} F^{l}$ to all 'trivial' normals, that is, normals $\xi$, such that $\langle A, \xi\rangle=0$, where $A$ is the image of the second fundamental form [Sp3, p. 361]. Or, equivalently, the first normal space is the linear hull of the image of the second fundamental form $\operatorname{span}\{\operatorname{Im} A\}$. The dimension of the first normal space will be called the point codimension of the surface $F^{l}$ at the point $Q$ (notation: $\operatorname{codim}_{p} F^{l}(Q)$ ).

THEOREM 3. A $C^{2}$ smooth surface is a developable surface if and only if its metric is flat and the point codimension is 1 everywhere.

The assumption on the point codimension is crucial as is shown by the following example.

The Clifford torus: This is the direct product of two circles $S^{1} \times S^{1} \subset E^{4}$. Its parametric equation is

$$
r=(\cos u, \sin u, \cos v, \sin v)
$$

Choosing the normals so that $n_{1}=(\cos u, \sin u, 0,0), n_{2}=(0,0, \cos v, \sin v)$ the matrices of the second fundamental form are

$$
A^{1}=\left(\left\langle r_{i j}, n_{1}\right\rangle\right)=\left(\begin{array}{cc}
-1 & 0 \\
0 & 0
\end{array}\right), \quad A^{2}=\left(\left\langle r_{i j}, n_{2}\right\rangle\right)=\left(\begin{array}{cc}
0 & 0 \\
0 & -1
\end{array}\right) ;
$$

the Gaussian curvature vanishes, the metric is flat; the surface is not even ruled (it is complete, compact);

$$
\operatorname{codim}_{p} F^{2}=2 \text { everywhere. }
$$

COROLLARY. Any $C^{2}$ smooth hypersurface $F^{l} \subset E^{l+1}$ without planar points possessing flat metric is a developable surface.

Certainly, this result is not new and we produce it only for the sake of completeness of the picture. 
PROOF OF THEOREM 3 (necessity). Due to the definition of a developable surface the point codimension of a developable surface equals 1 at every point, since there is only one non- trivial normal $A_{l l}$. The flatness of the metric follows from the Gauss equation.

PROOF OF THEOREM 3 (sufficiency). At a point $Q$ the second fundamental form $A$ can be reduced to the diagonal form with respect to the unique non-trivial normal. The condition of the metric flatness implies that the product of any two diagonal elements vanishes, whence all these elements are zero, except maybe one. The last one is not zero as $\operatorname{codim}_{p} F^{l}(Q)>0$. Therefore, $v=l-1$.

The next theorem shows that a surface of point codimension 1 is either a hypersurface or a developable surface. Or, in other words, for a surface $F^{l} \subset E^{n}$ with non-flat metric the condition $\operatorname{codim}_{p} F^{l} \equiv 1$ implies the existence of some $E^{l+1} \subset E^{n}$ containing the surface: $F^{l} \subset E^{l+1} \subset E^{n}$. For a rigorous statement one needs the following definition.

By the local codimension $\operatorname{codim}_{\mathrm{loc}} F^{l}(Q)$ of a surface $F^{l}$ at a point $Q$ we mean the difference between the dimension of the minimal Euclidean space, containing some neighbourhood of $Q$, and the dimension of the surface itself.

It is clear that

$$
\operatorname{codim}_{p} F^{l}(Q) \leq \operatorname{codim}_{\mathrm{loc}} F^{l}(Q) .
$$

THEOREM 4. If for all points $Q$ of a $C^{2}$ smooth surface $F^{l} \subset E^{n}$

$$
1=\operatorname{codim}_{p} F^{l}(Q)<\operatorname{codim}_{\mathrm{loc}} F^{l}(Q),
$$

then $F^{l}$ is a developable surface.

This theorem, in contradistinction to the previous and the subsequent ones, is not quite a characteristic property of a developable surface. But it can be easily made such by the following reformulation: a $C^{2}$ smooth surface $F^{l} \subset E^{n}$, which is not a local hypersurface (that is, the local codimension $>1$ ), is a developable surface if and only if for every point $Q \operatorname{codim}_{p} F^{l}(Q)=1$.

REMARK. This theorem was stated by Segre [Se] for an analytic $F^{2} \subset E^{n}$, although there is a gap in the proof. Adapting Segre's proof one can decrease the requirements on smoothness to $C^{2}$. We prefer to present here a short proof, employing the moving frame method, which requires $C^{4}$ smoothness.

ProOf OF THEOREM 4. As $F^{l} \subset E^{n}$ is $C^{4}$ smooth one can introduce on $F^{l}$ a $C^{2}$ orthonormal frame $\left\{e_{A}\right\}_{1}^{n}$ such that $\operatorname{span}\left\{e_{i}\right\}_{1}^{l}=T F^{l}$, and $e_{l+1}$ is the unique non-trivial 
normal. There is the expansion (3) for this frame. The condition of triviality of the other normals is

$$
h_{i}^{\beta}=0, \quad(1 \leq i \leq l, \quad l+2 \leq \beta \leq n) .
$$

The condition $\operatorname{codim}_{\mathrm{loc}} F^{l}>1$ means that there exists $\beta \in\{l+2, \ldots, n\}$ such that the torsion form $k_{l+1}^{\beta} \neq 0$. Without loss of generality one can assume

$$
k_{l+1}^{l+2} \neq 0 \text {. }
$$

The Codazzi-Mainardi equation for $h_{i}^{l+2}(1 \leq i \leq l)$ with (8) and (9) gives

$$
\begin{gathered}
0=d h_{i}^{l+2}+h_{j}^{l+2} \wedge \psi_{i}^{j}+\kappa_{\alpha}^{l+2} \wedge h_{i}^{\alpha}=\kappa_{l+1}^{l+2} \wedge h_{i}^{l+1} \\
(1 \leq i, j \leq l, \quad l+1 \leq \alpha \leq n) .
\end{gathered}
$$

Rotating the frame $\left\{e_{i}\right\}_{1}^{l}$ one can achieve $\kappa_{l+1}^{l+2}=k \cdot \omega^{l}$, where $k \neq 0$ is a realvalued function on $F^{l}$. For the new frame the equation (10) turns into $h_{i}^{l+1} \wedge \omega^{l}=0$ $(1 \leq i \leq l)$, that is, $h_{i}^{l+1}=A_{i l}^{l+1} \omega^{l}, A_{i j}^{l+1}=0(1 \leq i \leq l, 1 \leq j \leq l-1)$. Since the matrix $A^{l+1}$ is symmetric, $A_{i l}^{l+1}=A_{l i}^{l+1}=0(1 \leq i \leq l-1)$. Therefore, for the new frame

$$
h_{i}^{l+1}=0 \quad(1 \leq i \leq l-1), \quad h_{l}^{l+1}=A_{l l}^{l+1} \omega^{l},
$$

which precisely corresponds to the definition of a developable surface.

\section{Developable surfaces as affinely stable immersions of the flat metric}

A surface $F^{l} \subset E^{n}$ is called an affinely stable immersion of the flat metric, if for any affine transformation $g$ of $E^{n}$ the new surface $g\left(F^{l}\right)$ has flat metric.

The notion of a ' $G$-stable immersion' for different classes of metrics and for different groups $G$ was introduced in [Sh] and developed in [BSh].

THEOREM 5. A $C^{2}$ smooth surface without planar points is a developable surface if and only if it is an affinely stable immersion of the flat metric.

PROOF OF THEOREM 5 (necessity). From Theorem 1 a developable surface $F^{l}$ is $(l-1)$-ruled with tangent planes stable along the rulings. Both of these properties are invariant under affine (and even projective) transformations. The easiest way to show this is by stating the stability condition as follows: the plane tangent to $F^{l}$ at $Q$ is tangent to $F^{l}$ at an arbitrary point of the $(l-1)$-dimensional ruling passing through $Q$. Therefore, a developable surface $F^{l}$ under an affine transformation $g$ becomes a developable surface $g\left(F^{l}\right)$. And since any developable surface possesses the flat metric (Theorem 3 ), a developable surface is an affinely stable immersion of the flat metric. 
PROOF OF THEOREM 5 (sufficiency). Let $F^{l}$ be an affinely stable immersion of the flat metric. Let us introduce in the ambient space $E^{n}$ a Cartesian coordinate system. Subjecting $F^{l}$ to an affine transformation we will simultaneously subject the coordinate system to the same transformation. Then the radius vector of $F^{l}$ is unaffected, and only the scalar product between the basis vectors of the coordinate system changes. From this point of view the affine stability of $F^{l}$ can be conveyed as follows: for an arbitrary choice of the scalar product in the ambient $\mathbb{R}^{n}$, the induced metric of $F^{l}$ given by the Gauss equation, is flat.

We intend to show that this condition implies the strong degeneracy of the second fundamental form of $F^{l}$ : its relative nullity index is $l-1$. For our purpose it is sufficient to keep the freedom of choice of the scalar product only inside $T_{Q}^{\perp} F^{l}$, while $T_{Q}^{\perp} F^{l}$ can be considered as a fixed space; then the images of the second fundamental form $A_{i j}$ will be fixed vectors in $T_{Q}^{\perp} F^{l}$ (in the general case they are the orthogonal projections of the unmoving vectors $r_{i j} \in \mathbb{R}^{n}$ onto the moving space $T_{Q}^{\perp} F^{l}$ ). The final stage of the proof can be stated as the following algebraic lemma for $U=T_{Q} F^{l}$, $V=T_{Q}^{\perp} F^{l}$.

LEMMA. Let $U, V$ be finite-dimensional linear spaces ( $V$ with a scalar product $\langle\cdot, \cdot\rangle)$ and

$$
A: U \times U \rightarrow V
$$

be a non-vanishing symmetric bilinear map such that

$$
K(x, y)=\langle A(x, x), A(y, y)\rangle-\langle A(x, y), A(x, y)\rangle=0
$$

for any choice of scalar product in $V$. Then the codimension of the nullity space of $A$ in $U$ is 1 .

PROOF OF LEMMA. The major part of the proof is in showing that the image of $A$ is 1- dimensional ( $\left.\operatorname{codim}_{p} F^{l}(Q)=1\right)$ ). Then Theorem 3 completes the proof.

Step 1. Construction of a directing vector in $\operatorname{Im} A$. Since $A \not \equiv 0$, there exists $x \in U$ such that $A(x, \cdot) \neq 0$. Then $A(x, x) \neq 0$. Indeed, $A(x, x)=0$ implies $A(x, \cdot)=0$ from the Gauss equation (11). Therefore, we can take $A(x, x)$ as the required directing vector in $\operatorname{Im} A$.

Step 2. For all $y \in U$ and for $x$ fixed above $A(x, y) \| A(x, x)$. We assume $\operatorname{dim} V \geq 3$ (for $\operatorname{dim} V=1$ there is nothing to prove and for $\operatorname{dim} V=2$ the following argument can be used with $\alpha_{3}=\beta_{3}=0$ ). Let us take three linear independent vectors $\left\{e_{i}\right\}_{1}^{3}$ in $V$, whose linear hull contains the set $A(x, x), A(x, y), A(y, y)$ and such that $e_{1}=A(x, x)$. Then

$$
A(x, x)=e_{1},
$$




$$
\begin{aligned}
& A(x, y)=\alpha_{1} e_{1}+\alpha_{2} e_{2}+\alpha_{3} e_{3}, \\
& A(y, y)=\beta_{1} e_{1}+\beta_{2} e_{2}+\beta_{3} e_{3} .
\end{aligned}
$$

From (11)

$$
\begin{aligned}
0=K(x, y)= & e_{1} \cdot\left(\beta_{1} e_{1}+\beta_{2} e_{2}+\beta_{3} e_{3}\right)-\left(\alpha_{1} e_{1}+\alpha_{2} e_{2}+\alpha_{3} e_{3}\right)^{2} \\
= & \left(\beta_{1}-\left(\alpha_{1}\right)^{2}\right) e_{1} \cdot e_{1}-\left(\alpha_{2}\right)^{2} e_{2} \cdot e_{2}-\left(\alpha_{3}\right)^{2} e_{3} \cdot e_{3} \\
& +\left(\beta_{2}-2 \alpha_{1} \alpha_{2}\right) e_{1} \cdot e_{2}+\left(\beta_{3}-2 \alpha_{1} \alpha_{3}\right) e_{1} \cdot e_{3}-2 \alpha_{2} \alpha_{3} e_{2} \cdot e_{3} .
\end{aligned}
$$

Since this equality must be preserved under an arbitrary choice of the scalar product, all the coefficients of $e_{i} \cdot e_{j}$ vanish, which gives $\beta_{1}-\left(\alpha_{1}\right)^{2}=\alpha_{2}=\alpha_{3}=\beta_{2}=\beta_{3}=0$, that is, $A(x, y) \| e_{1}=A(x, x)$.

Step 3. For all $y, z \in \boldsymbol{U} \boldsymbol{A}(\boldsymbol{y}, z) \| \boldsymbol{A}(\boldsymbol{x}, \boldsymbol{x})$. Suppose $A(y, z)$ is not parallel to $A(x, x)$. Let $e_{1}=A(x, x), e_{2}=A(y, z)$. Then $A(x, y)=\alpha e_{1}, A(x, z)=\beta e_{1}$. From (11)

$$
\begin{aligned}
0 & =K(x, y+z) \\
& =K(x, y)+K(x, z)+2[A(x, x) \cdot A(y, z)-A(x, y) \cdot A(x, z)] \\
& =2\left[e_{1} \cdot e_{2}-\alpha \beta e_{1} \cdot e_{1}\right] .
\end{aligned}
$$

This equality can not be valid for an arbitrary choice of the scalar product. This contradicts the assumption $A(y, z) \nmid A(x, x)$.

\section{Planar points}

In this section we will consider developable surfaces with planar points, which consist of both points of rank 1 (the relative nullity index $v=l-1$ ) and planar points (of rank 0 , or $v=l$ ).

The geometric structure of such surfaces is quite different. A developable surface with planar points $F^{l} \subset E^{n}$ is no longer properly $(l-1)$ - ruled: it is glued together from pieces of $(l-1)$-ruled developable surfaces without planar points and pieces of $l$-dimensional planes. There is a pleasant feature, however: if a point $Q \in F^{l}$ has rank 1 , then there exists a unique $(l-1)$-dimensional ruling (with stable tangent plane) passing through $Q$ and all the points of this ruling have rank 1 as well [H, Lemma 3.1]. Therefore, the gluing together of plane and ruled pieces is always made along $(l-1)$-rulings. Theorem 1 can be reformulated as follows:

THEOREM 1'. A $C^{2}$ smooth surface $F^{l} \subset E^{n}$ is a developable surface with planar points if and only if $F^{l}$ is $(l-1)$-ruled in the sense described below and the tangent planes are stable along the rulings. 
Here, by an $(l-1)$-ruled surface we mean the natural foliation of the non-flat pieces and the possibility of foliation of the plane pieces. Therefore, in the definition of a $(l-1)$-ruled surface we have to drop the condition of uniqueness of the $(l-1)$-ruling passing through any point.

The sufficiency part of the theorem can be proved nearly without changes. In the necessity part, however, there occurs the following difficulty: in attempting to construct a 'good' frame (Step 1), the smoothness of this frame will be $C^{0}$ and not higher in the general situation-even for any increase (up to $C^{\infty}$ ) of the smoothness of the initial $F^{l}$. In other words, the direction of the rulings (even if it is well-defined) might be not $C^{1}$ smooth. This phenomenon has been scrutinized in [U2]. Because of it the rest of the proof (Step 2 and Step 3) becomes unusable. This obstacle can be avoided by synthetic means: if there are no planar points in the infinitesimal vicinity of the point investigated, then the direction of the ruling is $C^{2}$ smooth and one can use the previous proof; if there are planar points in the infinitesimal vicinity of the point, the required stability of the tangent planes can be obtained by means of continuity from the obvious stability of the tangent planes of the plane pieces.

The other theorems can be reformulated as follows:

THEOREM 2'. A $C^{2}$ smooth surface $F^{l} \subset E^{n}$ is a developable surface with planar points if and only if $F^{\prime}$ is $(l-1)$-ruled (in the new sense) and its metric is flat.

THEOREM 3'. A $C^{2}$ smooth surface is a developable surface with planar points if and only if its metric is flat and the point codimension is at most 1 everywhere.

COROLLARY'. Any $C^{2}$ smooth hypersurface $F^{l} \subset E^{l+1}$ possessing flat metric is a developable surface with planar points.

THEOREM 4'. If for all points $Q$ of a $C^{2}$ smooth surface $F^{l} \subset E^{n}$ the point codimension satisfies $\operatorname{codim}_{p} F^{l}(Q) \leq 1$ and $\operatorname{codim}_{10 c} F^{l}(Q)>1$, then $F^{l}$ is a developable surface with planar points.

THEOREM 5'. A $C^{2}$ smooth surface is a developable surface with planar points if and only if it is an affinely stable immersion of the flat metric.

The proofs of the theorems go through practically without changes: one merely needs to consider the planar and non-planar parts separately.

\section{Acknowledgement}

I would like to thank Prof. V. A. Zalgaller (St.-Petersburg University) who stimulated my interest in the smoothness of the objects investigated. I am grateful to 
Prof. H. Rubinstein and Prof. W. Neumann who gave me the opportunity to work at the University of Melbourne.

\section{References}

[A] M. A. Akivis, 'Focal images of surfaces of rank k', Izv. Vyssh. Uchebn. Zaved. Mat. 9 (1957), 9-19 (Russian).

[AR] M. A. Akivis and V. V. Ryzhkov, Many-dimensional surfaces of special projective types, Proceedings of IV all-union mathematical congress 2 (1964) pp. 159-164 (Russian).

[B] A. A. Borisenko, 'On complete parabolic surfaces in Euclidean space', Ukrain. Geom. Sb. 28 (1985), 8-19 (Russian).

[BSh] Yu. D. Burago and S. Z. Shefel', 'The geometry of surfaces in Euclidean spaces', in: Itogi Nauki i Tekhniki, Sovremennye problemy matematiki, Fundamental'nye napravleniya (eds. Yu. D. Burago and V. A. Zalgaller), Geometriya 348 (VINITI, Moscow, 1989) pp. 1-85. English transl. Encyclopaedia of Mathematical Sciences, vol. 48, Geometry 3 (Springer, 1992) $1-85$.

[CK] S. S. Chern and N. H. Kuiper, 'Some theorems on the isometric imbedding of compact Riemannian manifolds in Euclidean space', Ann. of Math. 50 (1952), 422-430.

[DG] M. Dajczer and D. Gromoll, 'Rigidity of complete Euclidean hypersurfaces', J. Diff. Geom. 31 (1990), $401-416$.

[FW] G. Fischer and H. Wu, 'Developable complex analytic submanifolds', Internat. J. Math. 6 (1995), 229-272.

[H] P. Hartman, 'On isometric immersions in Euclidean space of manifolds with non-negative sectional curvatures', Trans. Amer. Math. Soc. 115 (1965), 94-109.

[HN] P. Hartman and L. Nirenberg, 'On spherical image maps whose Jacobians do not change sign', Amer. J. Math. 81 (1959), 901-920.

[HW1] P. Hartman and A. Wintner, 'On the fundamental equations of differential geometry', Amer. J. Math. 72 (1950), 757-774.

[HW2] - 'On the asymptotic curves of a surface', Amer. J. Math. 73 (1951), 149-172.

[HC] D. Hilbert and S. Cohn-Vossen, Geometry and the imagination (Chelsea Publishing Company, New York, 1952).

[KN] S. Kobayashi and K. Nomizu, Foundations of differential geometry, vol. 2 (Interscience Publishers Inc., New York, 1969).

[R] V. V. Ryzhkov, 'On tangentially degenerate surfaces', Dokl. Akad. Nauk SSSR 135 (1960) pp. 20-22 (Russian).

[Se] C. Segre, 'Su una classe di superficie degl'iperspazî, legata colle equazioni lineari alle derivate parziali di $2^{0}$ ordine', Atti Acad. Torino 42 (1907), 559-591 (Italian).

[Sh] S. Z. Shefel', 'Geometric properties of embedded manifolds', Sibirsk. Mat. Zh. 26 (1985), 170-188; English transl. Siberian Math. J. 26 (1985), 133-147.

[Sp1] M. Spivak, A comprehensive introduction to differential geometry, vol. III (Publish or Perish, Inc., Berkeley, 1979).

[Sp2] - A comprehensive introduction to differential geometry, vol. IV (Publish or Perish, Inc., Berkeley, 1979).

[Sp3] - A comprehensive introduction to differential geometry, vol. V (Publish or Perish, Inc., Berkeley, 1979).

[TSh] V. A. Toponogov and S. Z. Shefel', 'Geometry of immersed manifolds', in: Encyclopaedia of Mathematics, 4 (Kluwer Academic Publ., Amsterdam, 1993) pp. 264-267. 
[U1] V. Ushakov, Riemannian manifolds and surfaces of constant nullity (Ph.D. Thesis, St.Petersburg University, 1993), (Russian).

[U2] _ 'Parametrization of developable surfaces by asymptotic lines', Bull. Austral. Math. Soc. 54 (1996), 411-421.

[U3] , 'Smoothness of the solution of trivial Monge-Ampère equation', Bull. Austral. Math. Soc. 56 (1997), 439-445.

[W] H. Wu, 'Complete developable submanifolds in real and complex spaces', Internat. J. Math. 6 (1995), 461-489.

[Ya1] N. N. Yanenko, 'The geometric structure of surfaces of small type', Dokl. Akad. Nauk SSSR (N.S.) 64 (1949), 641-644 (Russian).

[Ya2] - 'Some questions of the theory of imbedding of Riemannian metrics in Euclidean spaces', Uspekhi Matem. Nauk (N.S.) 8 (1953), 21-100 (Russian).

Department of Mathematics

The University of Melbourne

Parkville VIC 3052

Australia

e-mail: v.ushakov@ms.unimelb.edu.au 